Case Report

\title{
Coexistence of Juvenile-Like Polyp with Neurofibroma at the Gastroesophageal Junction in an Adult with Neurofibromatosis Type I
}

\author{
Namrah Aijaz, ${ }^{1}$ Peter Draganov, ${ }^{2}$ Atif Iqbal, ${ }^{3}$ and Xiuli Liu ${ }^{1}$ \\ ${ }^{1}$ Department of Pathology, University of Florida, Gainesville, FL, USA \\ ${ }^{2}$ Department of Gastroenterology, University of Florida, Gainesville, FL, USA \\ ${ }^{3}$ Department of Surgery, University of Florida, Gainesville, FL, USA \\ Correspondence should be addressed to Xiuli Liu; xiuliliu@ufl.edu
}

Received 11 July 2016; Accepted 1 November 2016; Published 12 January 2017

Academic Editor: Amit Agrawal

Copyright (C) 2017 Namrah Aijaz et al. This is an open access article distributed under the Creative Commons Attribution License, which permits unrestricted use, distribution, and reproduction in any medium, provided the original work is properly cited.

\begin{abstract}
A 23-year-old Caucasian male with Neurofibromatosis Type I (NF-I) was found to have a submucosal nodule at the gastroesophageal junction (GEJ) and underwent endoscopic submucosal dissection. Histological examination revealed two different lesions within the nodule. The dominant lesion was inflammatory/hyperplastic (juvenile-like) polyp with obliterative vasculopathy while the smaller lesion was a neurofibroma. Gastric/GEJ lesions in NF-I are very rare with only seven cases reported in the literature. Three cases of juvenile-like gastric polyps (located in the antrum, greater curvature, and fundus) have been reported in adult NF-I patients. An inflammatory polyp associated with a neurofibroma has only been described once in the pediatric literature but never in an adult. Our case is unique from those previously described in the literature due to the age of the patient, the presence of 2 histologically separate lesions in one endoscopically evident lesion, and the presence of obliterative vasculopathy in the juvenile-like polyp.
\end{abstract}

\section{Introduction}

Neurofibromatosis Type 1 (NF-I; von Recklinghausen disease) is the most common autosomal dominant hereditary disease, with an estimated birth incidence of approximately one in 3000 [1]. The disease manifestations vary greatly among affected patients and within families. The diagnosis of NF-I is clinical based on National Institutes of Health $(\mathrm{NIH})$ consensus guidelines which have not changed much since 1988 [2]. Gastrointestinal (GI) involvement by NFI varies widely from benign lesions and intermediate neoplasms to uncommon aggressive malignancies [3]. Of these, gastrointestinal stromal tumor (GIST) represents the most frequent GI manifestation affecting approximately a quarter of the patients $[3,4]$. Peripheral nerve sheath tumors such as neurofibroma, diffuse neurofibromatosis, and ganglioneuromatosis are much less common $[5,6]$. The incidence of mucosal nonadenomatous NF-I associated GI polyps is even more rare and varies on a spectrum ranging from hyperplastic polyps and inflammatory polyps to juvenile-like polyps.

Hyperplastic polyps in the gastroesophageal junction (GEJ) show a proliferation of hyperplastic gastric foveolartype epithelium (gastric) or squamous epithelium (distal esophageal) or a combination of the two with variable amounts of inflamed stroma [7]. Cases of inflammatory/hyperplastic polyp at the gastroesophageal junction (GEJ) have not been reported in adult patients with NF-1 although 3 cases of gastric inflammatory/hyperplastic polyps have been reported previously in NF-1 patients [8]. The rarity of these lesions and the application of endoscopic submucosal dissection (ESD) for lesion removal which allowed for en bloc removal with accurate histopathologic evaluation led us to report a case of an adult with a lesion containing an inflammatory/hyperplastic polyp and a neurofibroma and to perform a thorough review of the literature for similar cases. 

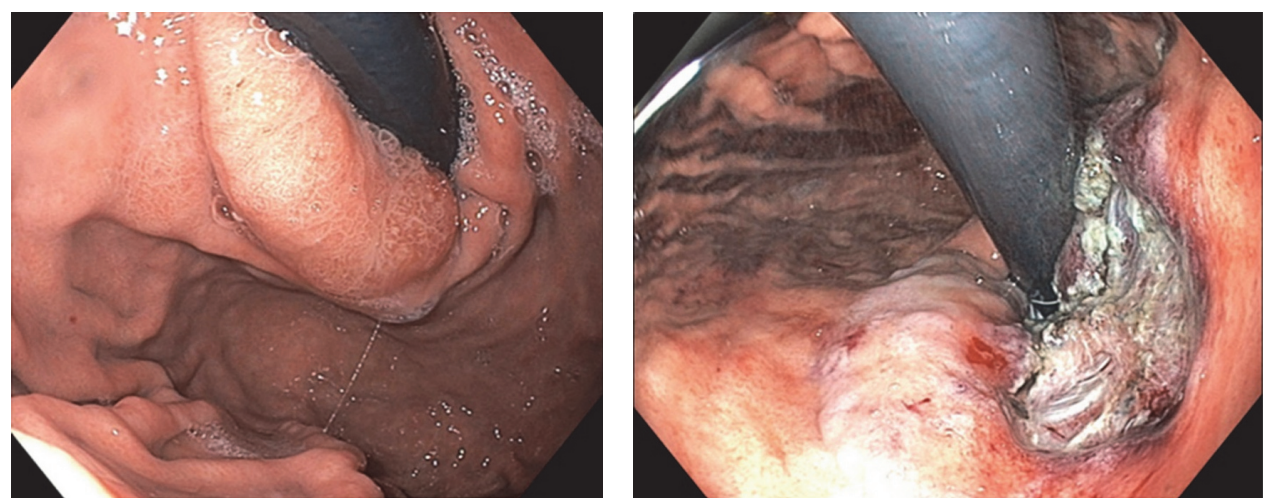

FIGURE 1: Endoscopic appearance before and after resection (retroverted view).

\section{Case Presentation}

A 23-year-old Caucasian male with a history of Neurofibromatosis Type I (NF-I) since birth, without any developmental delays, was evaluated for recurrent nausea, vomiting, and reflux esophagitis. He had a family history of NF-I on the paternal side. He had a history of multiple pigmented spots on the trunk with no change in the number or size of the lesions. He also complained of leg and buttock pain which was being managed by pain medication. He denied any other neurological symptoms such as numbness, weakness, or vision change. Physical examination revealed multiple caféau-lait spots, mainly distributed on the back and chest.

Lumbar spine MRI showed bilateral plexiform neurofibromas which were also present on a previous MRI few years ago. Colonoscopy was performed which was unremarkable without detectable polyps. Esophagogastroduodenoscopy (EGD) revealed a single well-defined $20 \times 10 \mathrm{~mm}$ subepithelial nodule at the GEJ. Endoscopic guided forceps biopsy revealed acute esophagitis with ulceration and granulation tissue without any neoplastic tissue identified. On endoscopic ultrasound, the lesion was hypoechoic originating from the submucosa without any deeper penetration. Ultrasoundguided fine needle aspiration (FNA) showed rare fragments of bland-appearing spindle cells, suspicious for a submucosal spindle cell lesion, but the tissue was not sufficient for further characterization.

Due to the submucosal location and uncertainty of the exact nature of the lesion, ESD was performed for en bloc removal. At the time of the ESD, en bloc resection was achieved and a 46 by $35 \mathrm{~mm}$ area involving $40 \%$ of the GEJ circumference was resected (Figures 1 and 2). The patient tolerated the procedure well with no adverse events.

Subsequent histologic examination of the nodule revealed two different lesions within the nodule. The dominant lesion consisted of hyperplastic gastric type mucosa with coiled elongated foveolae lined by mucin secreting cells (Figure 3). Mucus glands were present underneath surface epithelium admixed with edema, fibrosis, and nonspecific inflammation. Some of the glands were dilated and cystic. Several foci of obliterative vasculopathy were present in the deep portion of the lesion at the resection margin (Figure 4). These features were consistent with NF-1 associated inflammatory

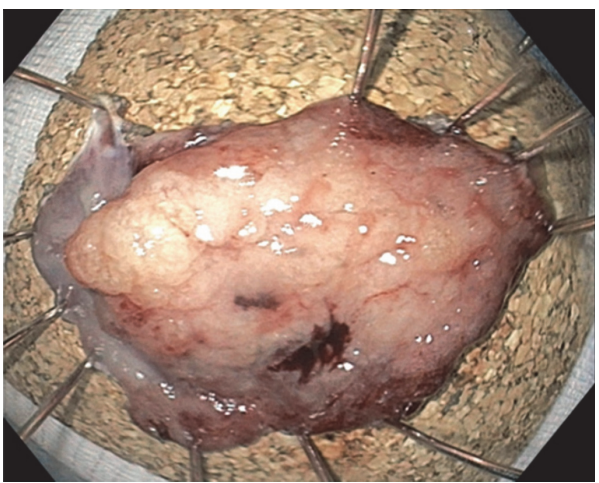

FIgURE 2: Lesion after endoscopic submucosal dissection.

or juvenile-like polyp. The smaller lesion consisted of a nonencapsulated lesion with bland spindle cell proliferation (Figures 5 and 6). The spindle cells have a wavering morphology and contain tapering nuclei. Occasional mast cells were seen admixed with collagen and fibroblasts with this lesion. No pleomorphism, necrosis, or mitosis was seen in this lesion. The spindle cells were positive for S-100 but negative for SMA, CD117, EMA, and CD34. Rare neurofilament positive nerve fibers were present within the lesion. These findings supported the diagnosis of a neurofibroma. The patient did well from the endoscopic resection and continues to symptomatically do better at one-month follow-up.

\section{Discussion}

NF-I is a multisystem genetic disorder that is commonly associated with cutaneous findings such as café-au-lait spots and axillary freckling, neurologic findings including both benign and malignant nervous system tumors, most notably benign neurofibromas, and orthopedic manifestations such as skeletal dysplasia. The frequency of gastrointestinal manifestations in NF-I is estimated to be between 11 and $25 \%$ [9] and they mainly include mucosal neurofibromas and gastrointestinal stromal tumors, although gastric carcinoids are also reported $[4,10]$. 


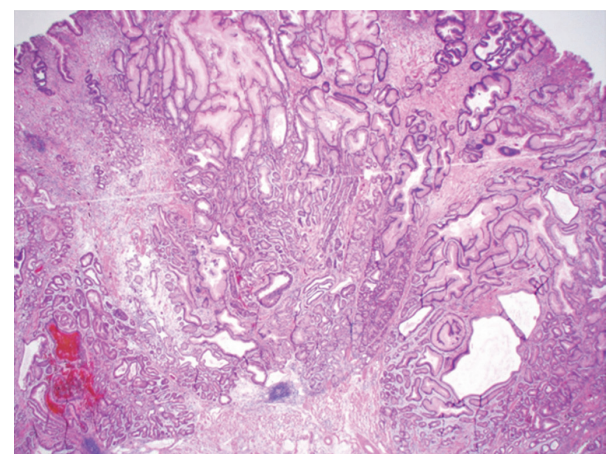

Figure 3: Hematoxylin and eosin stain shows hyperplastic gastric type mucosa with coiled elongated foveolae.

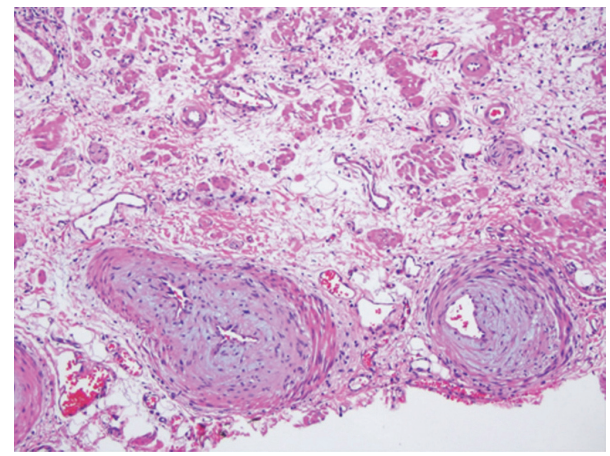

FIgURE 4: Hematoxylin and eosin stain shows obliterative vasculopathy at resection margin.

Inflammatory polyps (nonadenomatous, juvenile-like polyps) are uncommon in NF-I, occurring mostly in children, and although they have been reported in the literature they have not yet formally been included in the list of the disease manifestations [8]. Gastric/GEJ lesions are extremely rare with only seven cases reported in the literature for both pediatric and adult populations [11-14]. More specifically, only six cases of gastric/GEJ polyps were reported in NFI patients including three pediatric NF-1 patients with all polyps locating at the GEJ and three adult NF-I patients with all polyps in the stomach $[11,12]$. No GEJ juvenile-like polyps have been previously described in adult NF-I patients. Further, coexistence of juvenile-like polyp with neurofibroma has never been reported in an adult, although a single case of an inflammatory polyp associated with a neurofibroma has been described once in the pediatric literature [7].

Our case demonstrates a few important points. A thorough evaluation with standard techniques including EGD with biopsy and FNA and endoscopic ultrasound did not provide definitive diagnosis, but endoscopy with ESD was able to safely remove the lesion en bloc which provided the opportunity for complete lesion excision and comprehensive histopathologic evaluation. Furthermore, our case is unique because of the age of the patient, the presence of 2 histologically separate lesions in one endoscopically evident nodule,

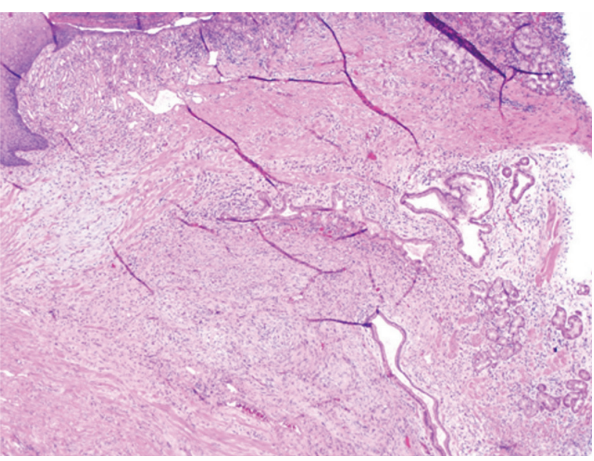

FIGURE 5: Hematoxylin and eosin stain: high power view of polyp showing dilated glands admixed with fibrosis and inflammation.

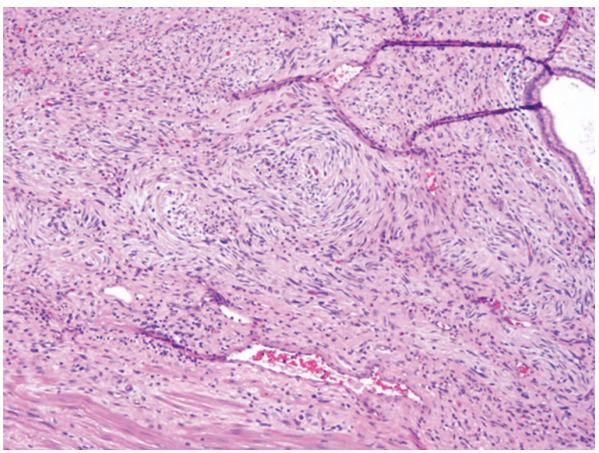

Figure 6: Hematoxylin and eosin stain shows bland spindle cell proliferation adjacent to polyp consistent with neurofibroma.

and the presence of obliterative vasculopathy in the juvenilelike polyp. To our knowledge, this is the first case of concurrence of GEJ juvenile-like polyp and neurofibroma in an adult NF-I patient. The etiology of juvenile-like (hyperplastic/inflammatory) polyp is not clear. However, the presence of significant obliterative vasculopathy in the current case as well as in one previously described inflammatory cecal polyp [8] suggests that ischemic injury with an exuberant regeneration may play a role in the pathogenesis of juvenilelike inflammatory/hyperplastic polyp in NF-1 patients. The clinical significance of these juvenile-like lesions in NF-I patients is not clear and the relationship of these lesions with the NF-I genotype needs further studies.

\section{Literature Review}

Hyperplastic/nonadenomatous gastrointestinal polyps in the setting of NF-I have been reported in 16 patients in the literature written in English language $[8,15]$. Of these, only 7 patients had gastric/GEJ lesions which are summarized in Table 1. Both pediatric and adult cases have been reported (3 pediatric, 4 adult) with a mean age of 29 years and a range of 1.5-69 years. A male predisposition is noted (6 males, 1 female). All patients presented with GI symptoms and/or chronic iron deficiency anemia, in cases with a detailed clinical history. Pediatric cases often presented with growth failure or abdominal symptoms. Within the stomach, 3 patients had 
TABLE 1: Literature review of all hyperplastic/inflammatory (juvenile-like) gastric/GEJ polyps in patients with Neurofibromatosis Type 1 $(n=7)$.

\begin{tabular}{ccccccccccc}
\hline & $\begin{array}{c}\text { Age } \\
\text { (years) }\end{array}$ & Author(s) & Gender & \multicolumn{3}{c}{ Polyps } & \multicolumn{2}{c}{ Other GI } & \multicolumn{2}{c}{ Other clinical } \\
features of NF-I
\end{tabular}

a single polyp while 4 patients had multiple polyps. Amongst the pediatric population, the lesions were located at the Z-line (1 patient), cardia (1 patient), and GEJ (1 patient) while, in the adult population, lesions were located at the greater curvature (1 patient), fundus (1 patient), antrum (2 patients), and distal esophagus (1 patient). Of note, one of the above adult patients had multiple polyps which were noted in both the fundus and the antrum. Other GI manifestations of NF-1 in patients listed in Table 1 included one or multiple small-bowel GISTs (two patients), duodenal somatostatinoma (one patient), duodenal hyperplastic-inflammatory polyp (one patient), interstitial cell of Cajal hyperplasia (one patient), cecal inflammatory polyp (one patient), and angiomatous colonic polyp (one patient).

Comprehensive histological description of the index lesion was available for five cases $[8,13,14]$. Three lesions showed primarily hyperplastic gastric foveolar-type epithelium without significant inflammatory infiltrate. A large antral lesion which was initially mistaken as a GIST due to diffuse hemorrhagic necrosis suggestive of an underlying vascular lesion with an extensive fibroblastic reaction and a few haphazardly distributed S100 positive nerve fibers in the lamina propria. Detailed examination did not reveal any underlying neurofibromatous or ganglioneuromatous elements. One polyp was predominantly inflammatory with some crypt distortion, hypervascularization with evidence of NF-I associated vasculopathy as suggested by thick walled vessels showing fibromyxoid intimal thickening, and a cellular stroma composed of bland- looking ovoid fibroblastic cells [8]. Dysplasia was not noted in any of these polyps. CMV testing was done in only one patient and was negative. Genetic analysis was not performed in any case; however a clinical diagnosis of NF-I was established in all cases. Hence, genotypic correlations for the development of hyperplastic/inflammatory polyp phenotype cannot be established.

When specifically looking at the three patients with GEJ lesions, two were pediatric, and polyp size ranged from 2 to $21 \mathrm{~mm}$ (mean of $13 \mathrm{~mm}$ ). All patients except the adult with the GEJ lesion had a preexisting confirmed clinical diagnosis of
NF-I based on the presence of two or more major NIH criteria $[8,11-14]$. The adult without a preexisting diagnosis had a family history of NF-I, underwent surgery for adhesions, was found to have a small-bowel GIST, and was subsequently diagnosed with NF-I along with presence of inflammatory polyps in the distal esophagus and the cecum [8].

\section{Competing Interests}

The authors declare that there are no competing interests regarding the publication of this paper.

\section{References}

[1] R. E. Ferner, "Neurofibromatosis 1 and neurofibromatosis 2: a twenty first century perspective," The Lancet Neurology, vol. 6, no. 4, pp. 340-351, 2007.

[2] "National Institutes of Health Consensus Development Conference Statement: neurofibromatosis. Bethesda, Md, USA, July 13-15, 1987," Neurofibromatosis, vol. 1, pp. 172-178, 1988.

[3] A. Agaimy, N. Vassos, and R. S. Croner, "Gastrointestinal manifestations of neurofibromatosis type 1 (Recklinghausen's disease): clinicopathological spectrum with pathogenetic considerations," International Journal of Clinical and Experimental Pathology, vol. 5, no. 9, pp. 852-862, 2012.

[4] M. Miettinen, J. F. Fetsch, L. H. Sobin, and J. Lasota, "Gastrointestinal stromal tumors in patients with neurofibromatosis 1 : a clinicopathologic and molecular genetic study of 45 cases," American Journal of Surgical Pathology, vol. 30, no. 1, pp. 9096, 2006.

[5] A. Agaimy, B. Märkl, J. Kitz et al., "Peripheral nerve sheath tumors of the gastrointestinal tract: a multicenter study of 58 patients including NF1-associated gastric schwannoma and unusual morphologic variants," Virchows Archiv, vol. 456, no. 4, pp. 411-422, 2010.

[6] K. Thway and C. Fisher, "Diffuse ganglioneuromatosis in small intestine associated with neurofibromatosis type 1," Annals of Diagnostic Pathology, vol. 13, no. 1, pp. 50-54, 2009.

[7] S. C. Abraham, V. K. Singh, J. H. Yardley, and T.-T. Wu, "Hyperplastic polyps of the esophagus and esophagogastric junction: 
histologic and clinicopathologic findings," American Journal of Surgical Pathology, vol. 25, no. 9, pp. 1180-1187, 2001.

[8] A. Agaimy, I.-M. Schaefer, L. Kotzina et al., "Juvenile-like (inflammatory/hyperplastic) mucosal polyps of the gastrointestinal tract in neurofibromatosis type 1," Histopathology, vol. 64, no. 6, pp. 777-786, 2014.

[9] I. Pinsk, O. Dukhno, A. Ovnat, and I. Levy, "Gastrointestinal complications of von Recklinghausen's disease: two case reports and a review of the literature," Scandinavian Journal of Gastroenterology, vol. 38, no. 12, pp. 1275-1278, 2003.

[10] U. Basile, G. Cavallaro, A. Polistena et al., "Gastrointestinal and retroperitoneal manifestations of type 1 neurofibromatosis," Journal of Gastrointestinal Surgery, vol. 14, no. 1, pp. 186-194, 2009.

[11] E. D. Palmer, Stomach Disease as Diagnosed by Gastroscopy, Kirnpton, London, UK, 1949.

[12] A. M. Hoare and S. G. Elkington, "Gastric lesions in generalized neurofibromatosis," British Journal of Surgery, vol. 63, no. 6, pp. 449-451, 1976.

[13] C. De Giacomo, R. Gullotta, P. Perotti, P. Bawa, M. Cornaggia, and R. Fiocca, "Hyperplastic esophagogastric polyps in two children with neurofibromatosis type 1," Journal of Pediatric Gastroenterology and Nutrition, vol. 18, no. 1, pp. 107-110, 1994.

[14] D. Grynspan, M. Lukacik, S. Madani, and J. Poulik, "Two hyperplastic esophagogastric polyps in a child with neurofibromatosis type 1 (NF-1)," Pediatric and Developmental Pathology, vol. 11, no. 3, pp. 235-238, 2008.

[15] K. Goto, T. Hirosaki, and M. Masubuchi, "Neurofibromatosis Type 1-associated inflammatory polyp of the gastrointestinal tract: clinicopathologic analysis of a surgically resected case," International Journal of Surgical Pathology, 2016. 


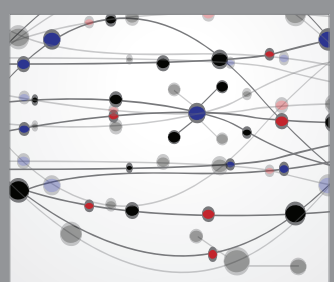

The Scientific World Journal
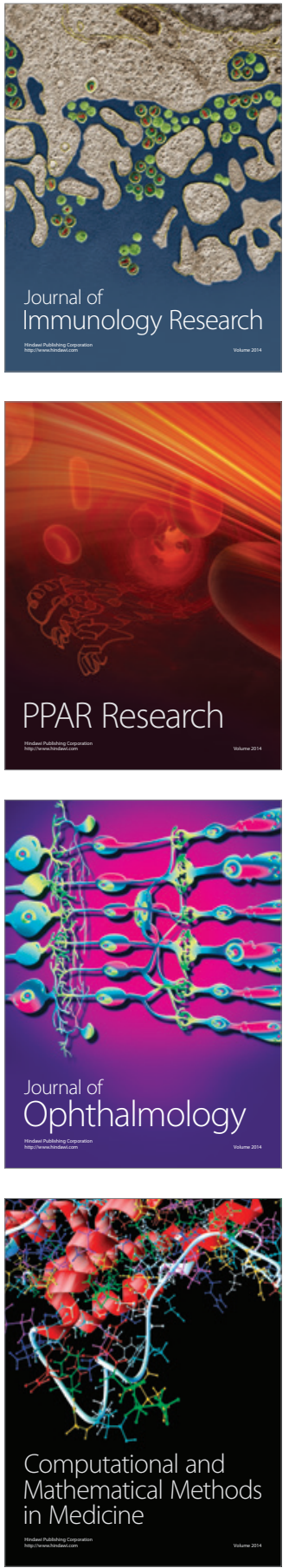

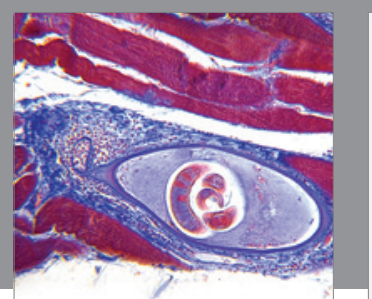

Gastroenterology Research and Practice
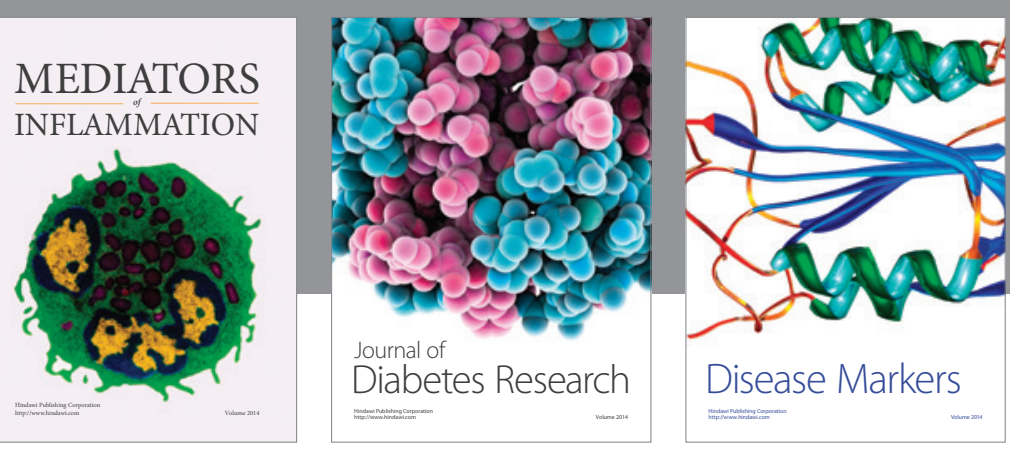

Disease Markers

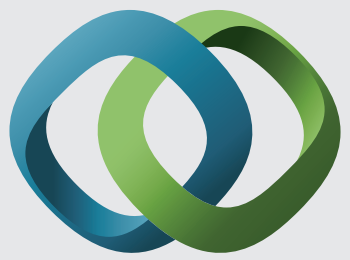

\section{Hindawi}

Submit your manuscripts at

https://www.hindawi.com
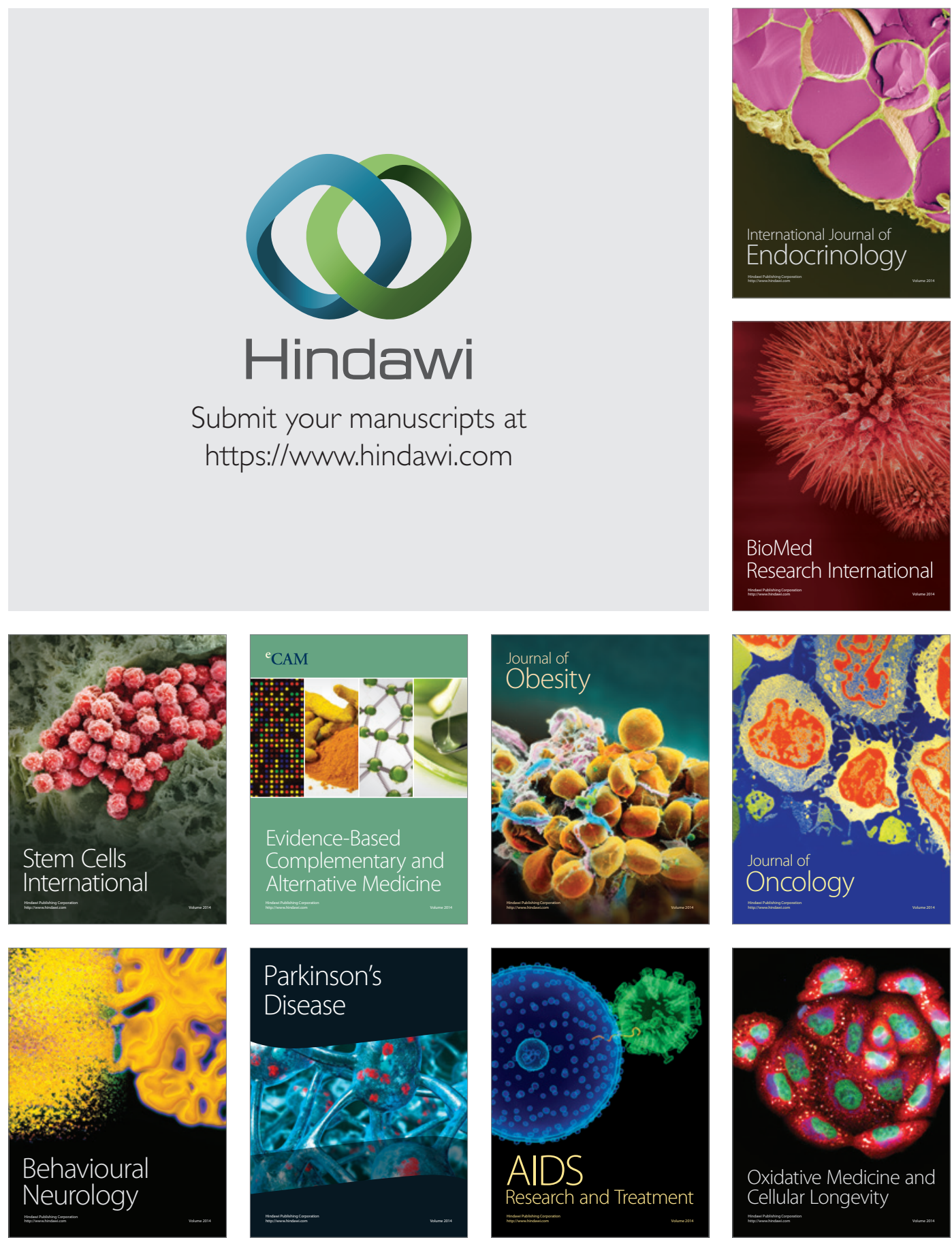